\title{
Diagnostic biopsy of the motor nerve to the gracilis muscle
}

\section{Clinical pearl}

\section{Kamel Abouzahr, M.D., Dale J. Lange, M.D., Norman Latov, M.D., Ph.D., Marcelo Olarte,} M.D., Lewis P. Rowland, M.D., Arthur P. Hays, M.D., and Massimo Corbo, M.D.

Departments of Surgery, Neurology, and Pathology, Columbia University College of Physicians and Surgeons, New York, New York; and H. S. Raffaelle, Clinica Neurologica, Universita di Milano, Milano, Italy

A biopsy specimen of the motor nerve to the gracilis muscle was obtained in 151 patients to aid in the diagnosis of neuromuscular disorders. The procedure is simple, safe, and has low morbidity and complication rates. The surgical technique is described. Indications for the use of this technique are motor neuropathy, motor neuron disease, Guillain-Barre syndrome, and mixed neuropathy with prominent motor symptoms.

Key Words * motor nerve * gracilis muscle

Peripheral nerve biopsy is useful in the diagnosis and management of neuromuscular disorders. The nerve selected should be affected by the disease process, display a constant anatomy, be of adequate size, and one which will produce a minimal deficit.[4]

Because of these requirements, the sural nerve in the leg has become the preferred biopsy site. $[1,5,10,12]$ Unfortunately, its histopathology is generally normal in diseases that preferentially affect the motor fibers such as pure motor neuropathy, motor neuron disease, and occasionally in sensorimotor neuropathy. In 1991, biopsy of the motor nerve to the gracilis muscle was developed to allow histopathological analysis in these conditions.

The purpose of this paper is to describe the anatomy, indications, contraindications, technique, surgical results, and complications of motor nerve biopsy of the gracilis muscle.

\section{ANATOMY OF THE GRACILIS MUSCLE AND SURROUNDING STRUCTURES}

The gracilis muscle is a superficial muscle that is located on the medial aspect of the thigh. It arises from the pubis bone and inserts into the medial tibial condyle. It is a weak adductor of the thigh, but it also aids in knee flexion and internal rotation. Its blood supply is derived primarily from the medial femoral circumflex artery and vein. Because its action overlaps that of stronger muscles, it is considered expendable. That has made it a useful muscle in reconstruction of rectal sphincter, vagina, and perineal wounds, as well as in free tissue transfer for correction of facial paralysis and restoration of digital flexion. $[6-9,11]$ 
The obturator nerve is derived from the L2-4 roots, emerges from the obturator foramen, and divides into two branches at the lateral border of the adductor brevis. The posterior branch passes deep to the adductor brevis and innervates the adductor magnus. The anterior branch innervates the adductor brevis and then proceeds medially to end in three terminal branches. The most superficial one innervates the adductor longus. An intermediate branch, the medial cutaneous nerve of the thigh, provides sensibility to a skin territory in the distal and medial aspect of the thigh. Frequently, sensory branches from the femoral nerve supply the same skin territory. The last branch joins the medial femoral circumflex vessels at the hilum of the gracilis and innervates it (Fig. 1).

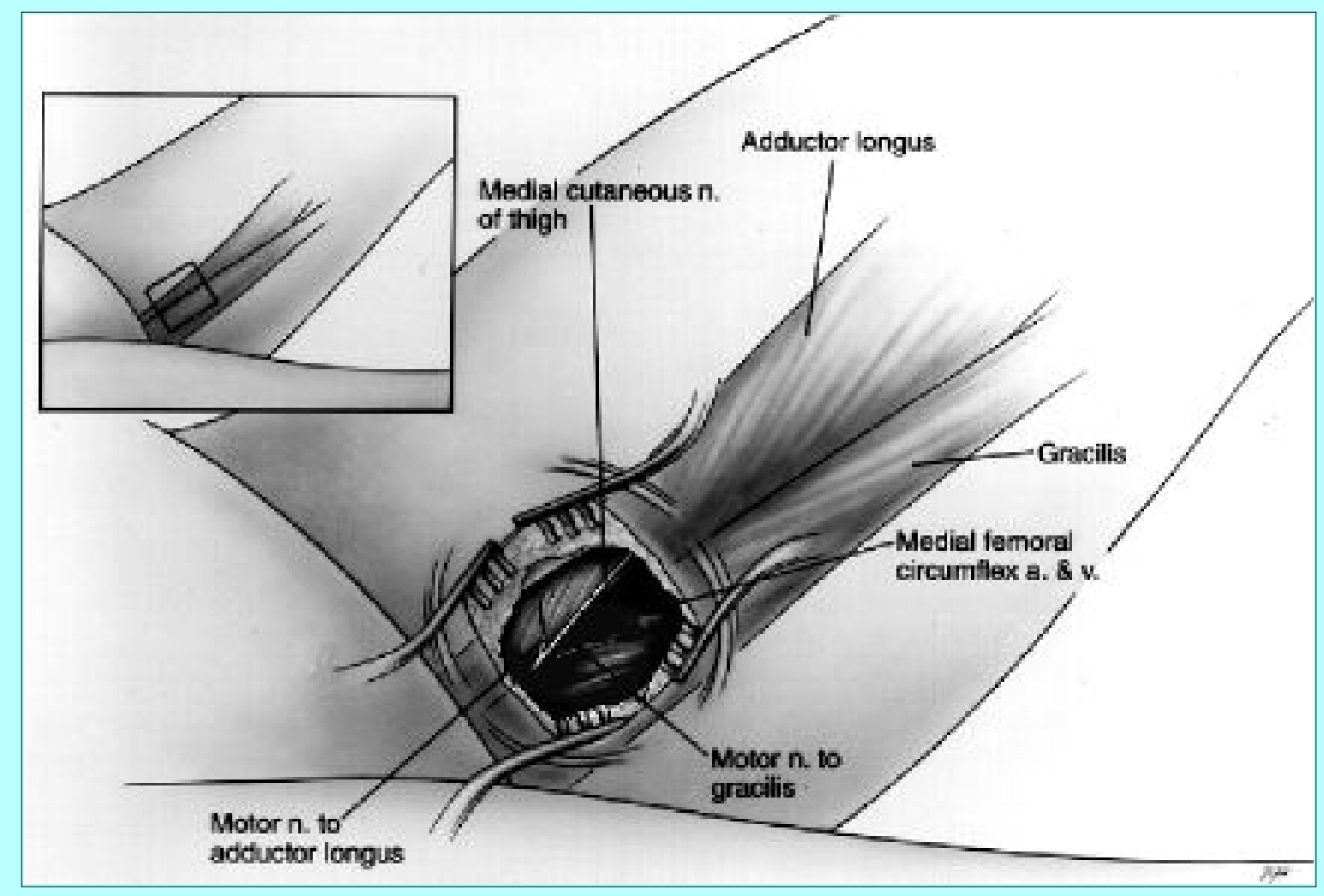

Fig. 1. Artist's illustration demonstrating the relationship between the terminal branches of the anterior branch of the obturator nerve and gracilis muscle. a. $=$ artery; $\mathrm{n} .=$ nerve; $\mathrm{v} .=$ vein.

\section{INDICATIONS AND CONTRAINDICATIONS FOR BIOPSY}

Biopsy of the motor nerve of the gracilis muscle is indicated in neuromuscular diseases that affect the thigh adductor musculature. These include pure motor neuropathy, sensorimotor neuropathy with predominant motor symptoms, Guillain-Barre syndrome, and motor neuron disease. Obtaining a biopsy specimen may be particularly advantageous in differentiating motor neuropathy from motor neuron disease; however, it is contraindicated in patients with coagulopathy or active perineal infections. Gracilis muscle biopsy is technically difficult to perform in patients with massive obesity and adduction contracture of the thigh.

\section{BIOPSY TECHNIQUE}

The patient is placed supine with the thigh abducted and externally rotated approximately $60^{\circ}$ (Fig. 1). An imaginary line from the pubis bone to the medial tibial condyle is outlined. Using a Doppler probe, the location of the medial femoral circumflex artery along that line is determined. Usually, it is located 
three- to four-finger breadths distal to the pubis bone. A 6-cm longitudinal incision is made over that point after infiltration with local anesthetic. The dissection proceeds to the intermuscular plane between the adductor longus anteriorly and the gracilis posteriorly. The motor nerve of the gracilis is seen to proceed from proximal-lateral to distal-medial at approximately a $45^{\circ}$ angle to the long axis of the limb. It penetrates the deep lateral muscle surface at its hilum. The nerve is 2 to $3 \mathrm{~mm}$ in width; a segment measuring approximately $4 \mathrm{~cm}$ can usually be obtained. Injury to the motor nerve of the adductor longus muscle must be avoided. If a sensory nerve biopsy is also warranted, the medial cutaneous nerve of the thigh, which measures approximately $1 \mathrm{~mm}$ in width, is dissected from the undersurface of the adductor longus and a biopsy specimen is obtained (Fig. 1).

\section{PATIENT POPULATION}

Between 1991 and 1996, 152 gracilis muscle motor nerve biopsies were obtained in 151 patients at Columbia-Presbyterian Medical Center in New York. There were 84 men and 67 women. Their ages ranged from 21 to 82 years (average 59 years). Right-sided biopsies were obtained in 72 patients, left-sided biopsies in 78 patients, and both sides in one patient. A gracilis muscle biopsy was obtained in each instance except in four cases, as planned preoperatively. Three biopsies were obtained in the adductor longus, medial gastrocnemius, and deltoid muscles instead. In the patient in whom bilateral biopsies were obtained, only a contralateral motor nerve biopsy was obtained during the second procedure.

\section{RESULTS}

The motor nerve was present in all patients. The medial femoral cutaneous nerve was present in 101 patients $(67 \%)$ and absent in 50 patients (33\%). Surgical complications included cellulitis in one (0.66), seroma in one, and a hematoma and seroma in one patient who developed coagulopathy attributable to high-dose steroid therapy. One patient suffered pulmonary aspiration postoperatively due to weakness of the swallowing musculature caused by motor neuron disease, which was not related to perioperative medications. Two patients $(1.3 \%)$ required reoperation to drain a fluid collection in the medial thigh; both healed uneventfully. The patient with cellulitis responded to oral antibiotic therapy and healed without sequelae.

\section{DISCUSSION}

Biopsy of the motor nerve to the gracilis muscle is a simple procedure. It has an acceptably low complication rate. Although the majority of patients already had weakness caused by their underlying disease, none complained of additional weakness or sensory loss as a result of the biopsy. Deutinger, et al.,[3] examined 42 patients in whom the gracilis muscle was used in reconstruction. In their series, there was an average decrease in thigh adduction strength of $11 \%$ and an area of hypesthesias in $40.5 \%$ of the patients; however, none of those patients noticed these changes.

Motor neuropathy and motor neuron disease in patients presenting with weakness may be difficult to differentiate on clinical grounds alone. Motor nerve analysis may provide an important distinction between these two diseases. Preliminary data from our institution disclose the usefulness of such analysis [2]. In addition, better understanding of the underlying pathophysiological processes may have diagnostic and therapeutic implications in the future.

\section{CONCLUSIONS}


The motor nerve of the gracilis muscle has constant anatomy, is dispensible, and its biopsy leaves the patient with minimal morbidity and disability. Its analysis could reveal important pathophysiological mechanisms in neuromuscular diseases.

\section{Acknowledgments}

The authors would like to thank Drs. David Chiu, David Younger, Saud Sadiq, Robert Lovelace, Robert De Napoli, Eugenia Gamboa, Douglas Kargman, and Frank Petito for their support of this work.

\section{References}

1. Asbury AK, Connolly ES: Sural nerve biopsy. Technical note. J Neurosurg 38:391-392, 1973

2. Carbo M, Abouzahr MK, Latov N, et al: Motor nerve biopsy studies in motor neuropathy and motor neuron disease. Muscle Nerve (In press, 1997)

3. Deutinger M, Kuzbari R, Paternostro-Sluga T, et al: Donor-site morbidity of the gracilis flap. Plast Reconst Surg 95:1240-1244, 1995

4. Dyck PJ, Lofgren EP: Method of fascicular biopsy of human peripheral nerve for electrophysiologic and histologic study. Mayo Clin Proc 41:778-784, 1966

5. Dyck PJ, Lofgren EP: Nerve biopsy. Choice of nerve, method, symptoms, and usefulness. Med Clin North Am 52:885-893, 1968

6. Graham JB: Vaginal fistulas following radiotherapy. Surg Gynecol Obstet 120:1019-1030, 1965

7. Harii K, Ohmori K, Torii S: Free gracilis muscle transplantation with microneurovascular anastomoses for the treatment of facial paralysis. A preliminary report. Plast Reconst Surg 57:133-143, 1976

8. Manktelow RT, McKee NH: Free muscle transplantation to provide active finger flexion. J Hand Surg (Am) 3:416-426, 1978

9. McCraw JB, Massey FM, Shanklin KD, et al: Vaginal reconstruction with gracilis myocutaneous flaps. Plast Reconst Surg 58:176-183, 1976

10. Moss JP, Meckler RJ, and Moss WE: Consistent effective technique for muscle and nerve biopsy. Am J Surg 138:736-737, 1979

11. Pickrell K, Masters F, Giorgade N, et al: Rectal sphincter reconstruction using gracilis muscle transplant. Plast Reconst Surg 13:46-55, 1954

12. Pollock M, Nukada H, Taylor P, et al: Comparison between fascicular and whole sural nerve biopsy. Ann Neurol 13:65-68, 1983

Manuscript received October 29, 1996.

Accepted in final form November 13, 1996.

Address reprint requests to: M. Kamel Abouzahr, M.D., 161 Fort Washington Avenue, New York, New York 10032. 
